Pacific Journal of Mathematics

CLASSIFICATION OF CLOSED SETS OF ATTAINABILITY IN 


\title{
CLASSIFICATION OF CLOSED SETS OF ATTAINABILITY IN THE PLANE
}

\author{
JAN M. GRONSKI
}

It is proved that under certain mild restrictions every closed set of attainability of a planar control system with piecewise constant controls is either the entire plane or homeomorphic to one of the following: a closed half plane, a closed disk or the complement of an open disk.

Let $D=\left\{X^{i}\right\}$ be a family of $C^{\infty}$ vector fields on $\boldsymbol{R}^{2}$. We say that a continuous mapping $\gamma:[a, b] \rightarrow \boldsymbol{R}^{2}, a<b$, is an integral curve of $D$ if there exists a finite partition of the interval $[a, b]: a=t_{0}<$ $t_{2}<\cdots<t_{n}=b$ such that $\gamma$ restricted to $\left[t_{j}, t_{j+1}\right]$ is an integral curve of some vector field $X^{i_{j}} \in D$. Let $x_{0}$ and $x_{1}$ be points of $\boldsymbol{R}^{2}$. We say that $x_{1}$ is attainable from $x_{0}$ (by means of $D$ ) if there exists an integral curve of $D, \gamma:[a, b] \rightarrow \boldsymbol{R}^{2}$ such that $\gamma(a)=x_{0}$ and $\gamma(b)=x_{1}$. Notice that we can always assume $a=0 ; \gamma(a)$ and $\gamma(b)$ are called endpoints of $\gamma$ and we set $\operatorname{Im} \gamma=\gamma([a, b])$. Let $A(x)$ denote the set consisting of $x$ and of all the points $y \in R^{2}$ which are attainable from $x$ by means of $D$. Similarly we denote by $A_{N}(x)$ the set consisting of $x$ and of all the points $y \in R^{2}$ attainable from $x$ by means of $D_{N}$, where $D_{N}=\{Y:-Y \in D\}$.

The sets $A(x), x \in R^{2}$ determined by a given family $D$ of vector fields on $\boldsymbol{R}^{2}$ can be viewed as the sets of attainability of a control system on $\boldsymbol{R}^{2}$, with discrete control space $D$ and piecewise constant controls taking values in $D$ (see Lobry [5]). By using the standard terminology from control theory, an integral curve $\gamma$ of $D$ (or the control defining it) is said to steer $x_{0}$ to $x_{1}$ if $x_{1}$ is attainable from $x_{0}$ via $\gamma$.

Families of vector fields on manifolds and their sets of attainability have recently been investigated by various authors Gerbier [2], Gronski [3].

Let $L$ denote the Lie subalgebra generated by the elements of $D$ in the algebra of all $C^{\infty}$ vector fields on $R^{2}$ and $L(x)$ be the space determined by $L$ in the tangent space at $x \in R^{2}$. We write the symbol $\mathrm{Cl} A$ to denote the closure of a set $A$, Int $A$ for its interior and $\partial A$ for its boundary.

We will use the following known result (see e.g., Krener [4] or Lobry [5]).

Theorem 1. If $\operatorname{dim} L(x)=2$, then $x \in \mathrm{Cl}(\operatorname{Int} A(x))$.

We prove first several auxiliary results. 
DEFINITION 2. Let $\gamma_{1}, \gamma_{2}$ be nonconstant integral curves of $D$. We say that $\gamma_{1}$ and $\gamma_{2}$ are distinct if $z \in \operatorname{Im} \gamma_{1} \cap \operatorname{Im} \gamma_{2}$ implies that $z$ is an endpoint of $\gamma_{1}$ and $\gamma_{2}$.

LEMMA 3. Let $\gamma$ be an integral curve of $D$ steering $x$ to $\partial A(x)$. Then $\operatorname{Im} \gamma \subseteq \partial A(x)$. (See Roxin [6].)

Proof. Suppose that $\gamma:[0, a] \rightarrow \boldsymbol{R}^{2}$ is an integral curve corresponding to some control $u$, such that $\gamma(0)=x$ and $\gamma(\alpha) \in \partial A(x)$. Assume that there exists a $t, 0 \leqq t<a$ with the property that $\gamma(t) \in \operatorname{Int} A(x)$. Notice that the corresponding control $\left.u\right|_{[t, a]}$ steers points near $\gamma(t)$ to points near $\gamma(a)$ and that this mapping is a local diffeomorphism at $\gamma(t)$. Thus $\gamma([t, a])$ is contained in Int $A(x)$, which is impossible.

An equivalent formulation of this lemma is that no point in the Int $A(x)$ can be steered to $\partial A(x)$.

Proposition 4. Let $x \in \boldsymbol{R}^{2}$. There are at most two nonconstant distinct integral curves of $D$ steering $x$ to $\partial A(x)$.

Proof. Let $u_{i}, i=1,2,3$ be three controls and let $\gamma_{i}: J_{i} \rightarrow R^{2}$, $i=1,2,3$, be the corresponding integral curves of $D$ starting at $x$. Suppose that each $\gamma_{i}$ steers $x$ to $\partial A(x)$ and that the $\gamma_{i}$ are pairwise distinct.

The existence of two nonconstant distinct integral curves of $D$ steering $x$ to $A(x)$ implies that $x \in \mathrm{Cl}(\operatorname{Int} A(x))$. This follows easily from continuity properties of flows of vector fields.

Let $\Gamma_{i}=\operatorname{Im} \gamma_{i}$. Choose an open ball $B$ about $x$ such that $B \backslash\left(\Gamma_{1} \cup \Gamma_{2} \cup \Gamma_{3}\right)$ has three connected components. Since $x \in \mathrm{Cl}(\operatorname{Int} A(x))$ we can assume that one of these components, say $C$, intersects Int $A(x)$ and that $x \in \mathrm{Cl}(\operatorname{Int} A(x) \cap C)$. It is clear that there exists $i$, say $i=1$, such that $\Gamma_{1} \cap \mathrm{Cl} C=\{x\}$. It follows that the boundary of $C$ contains parts of $\Gamma_{2}$ and $\Gamma_{3}$. Let $y \in \Gamma_{1} \cap B, y \neq x$ and $y=\gamma_{1}(t)$. Let $V$ be a neighborhood of $y$ such that $V \cap \mathrm{Cl} C=\varnothing$. Then $\left.u_{1}\right|_{[0, t]}$ steers a sufficiently small neighborhood of $x$ into $V$ without leaving $B$. As we have already noticed in Lemma 2 , this induces a local diffeomorphism at $x$. In particular, there are points in $C \cap \operatorname{Int} A(x)$ which are steered to a point in another component of $B \backslash\left(\Gamma_{1} \cup \Gamma_{2} \cup \Gamma_{3}\right)$. But then the corresponding integral curves of $D$ intersect $\partial A(x)$ which contradicts Lemma 2.

Note that this proof established also that sufficiently small ball about $x$ is separated by $\partial A(x)$ into exactly two components.

The next result is obtained by obvious modifications of Proposition 4 . 
Corollary 5. Let $\operatorname{dim} L(x)=2$ and $y \in A(x) \cap \partial A(x)$. Then $y$ is an endpoint of at most two distinct integral curves of $D$ contained in $\partial A(x)$.

In other words $\partial A(x)$ has no "branch points." The hypothesis $\operatorname{dim} L(x)=2$ could be replaced by a weaker assumption $x \in \mathrm{Cl}(\operatorname{Int} A(x))$. Simple examples show that without this assumption Corollary 5 is not true.

Let us now summarize what has been established so far. We know that there are at most two distinct integral curves steering $x$ to $\partial A(x)$ and neither of them "branches" while remaining in $\partial A(x)$. If they meet the union of their images constitutes the boundary of $A(x)$. Let us show what happens if they do not meet.

LemMa 6. If $y \in \partial A_{N}(x)$, then $A(y) \cap \operatorname{Int} A_{N}(x)=\varnothing$.

Proof. Suppose that there exists a point $z \in A(y) \cap \operatorname{Int} A_{N}(z)$. Then $y \in A_{N}(z) \subseteq \operatorname{Int} A_{N}(x)$ by the remark immediately following Lemma 3. But $y \in \partial A_{N}(x)$.

Lemma 7. Let $\alpha:[0, \alpha] \rightarrow \boldsymbol{R}_{2}$ be a homeomorphism onto $\operatorname{Im} \alpha_{1}$ and let $X^{1}$ be an arbitrary $C^{1}$ vector field on $\boldsymbol{R}^{2}$, with $X^{1}\left(\alpha^{1}(0)\right) \neq 0$. Let $t_{1}>0$ be such that $X_{t}(z) \notin \operatorname{Im} \alpha^{1}$ for all $z \in \operatorname{Im} \alpha$ and all $0<t \leqq t_{1}$. Then the mapping $H:[0, a] \times\left[0, t_{1}\right] \rightarrow \boldsymbol{R}^{2}$ defined by

$$
H^{1}(s, t)=X_{t}^{1}\left(\alpha^{1}(s)\right)
$$

is a homeomorphism onto its image.

The proof is straightforward and uses only existence, uniqueness and continuous dependence on the change of initial points of integral curves of $X^{1}$.

Let $\alpha^{1}$ be as above and let $\alpha^{2}(s)=H^{1}\left(\alpha^{1}(s), t_{1}\right)$. Assume that $X^{2}$ is a $C^{1}$ vector field on $R^{2}$ with $X^{2}\left(\alpha^{2}(0)\right) \neq 0$. Let $t_{2}>0$ be such that

$$
X_{t}^{2}(z) \notin H^{1}\left([0, a] \times\left[0, t_{1}\right]\right)
$$

for all $z \in \operatorname{Im} \alpha^{2}$ and $0<t \leqq t_{2}$. Let $H^{2}:[0, a] \times\left[0, t_{1}+t_{2}\right] \rightarrow \boldsymbol{R}^{2}$ by given by

$$
H^{2}(s, t)= \begin{cases}H^{1}(s, t) & t \leqq t_{1} \\ X_{t-b}^{2}\left(H^{1}\left(s_{1}, t_{1}\right)\right) & t>t_{1} .\end{cases}
$$

Clearly $H^{2}$ is a homeomorphism onto its image. It is also clear that given a collection $X^{1}, \cdots, X^{n}$ of vector fields satisfying appropriate conditions and a curve $\alpha^{1}$ we could construct inductively a sequence of homeomorphisms $H^{k}$ from some rectangle into $\boldsymbol{R}^{2}$. More precisely, 
let $c_{k-1}=\sum_{i=1}^{k-1} t_{i}$ and let $\alpha^{k}(s)=H^{k-1}\left(\alpha^{k-1}(s), c_{k-1}\right)$. Assume that the collection $X^{1}, \cdots, X^{n}$ satisfies the following properties:

1. $X^{1}$ satisfies the hypothesis of Lemma 7.

(*) 2. $X^{k}\left(\alpha^{k}(0)\right) \neq 0$.

3. $X_{t}^{k}(z) \notin H^{k-1}\left([0, a] \times\left[0, c_{k-1}\right]\right)$ for all $z \in \operatorname{Im} \alpha^{k}$ and $0<t \leqq t_{k}$. Then $H^{k}:[0, a] \times\left[0, c_{k}\right] \rightarrow \boldsymbol{R}^{2}$ defined by

$$
H^{k}(s, t)= \begin{cases}H^{k-1}(s, t) & t \leqq c_{k-1} \\ X_{t-c_{k-1}}^{k}\left(H^{k-1}\left(s, c_{k-1}\right)\right) & t>c_{k-1}\end{cases}
$$

is a homeomorphism for $1 \leqq k \leqq n$.

Let $x$ be an arbitrary point in $\boldsymbol{R}^{2}$ and let $z \in \partial A(x)$. Assume that $u$ is a control steering $z$ to a point $y \in \partial A(x)$. Moreover let

$$
y=X_{t_{n}}^{n} X_{t_{-1}}^{n-1} \cdots X_{t_{i-1}}^{1}(z),
$$

where $X^{i} \in D$ and are such that

$$
X^{i}\left(X_{t_{i-1}}^{i-1} \cdots X_{t_{1}}^{1}(z)\right) \neq 0
$$

for all $i \geqq 1$. Finally let $\alpha:[0, a] \rightarrow A(x)$ be a homeomorphism onto $\operatorname{Im} \alpha$ such that

$$
\begin{aligned}
& \alpha(0)=z, \\
& \alpha(t) \in \operatorname{Int} A(x) \quad 0<t \leqq a .
\end{aligned}
$$

Lemma 8. If $\alpha$ is sufficiently small then $\alpha, X^{1}, \cdots, X^{n}$ satisfy conditions $(*)$.

Proof. It follows immediately that $\operatorname{Im} X_{t}^{1}(\alpha(0)) \cap \operatorname{Im} \alpha=\alpha(0)=z$. Since $X^{1}$ is $C^{1}$ and $\left[0, t_{1}\right]$ is compact, it follows from the continuous dependence on the change of initial conditions that for sufficiently small $a, X_{t}^{1}(z) \notin \operatorname{Im} \alpha$ for $0<t \leqq t_{1}$, and $z \in \operatorname{Im} \alpha$. Thus

$$
H:[0, a] \times\left[0, t_{1}\right] \longrightarrow A(x)
$$

defined as before is a homeomorphism. Note that $H$ restricted to $\left[0, a^{1}\right] \times\left[0, t_{1}\right]$ where $a^{1} \leqq a$ is still a homeomorphism. One can now show by induction, repeating the same argument as above, that the lemma holds.

Lemma 9. Let $\operatorname{dim} L(x)=2$ and let $\gamma:[0, a] \rightarrow \boldsymbol{R}^{2}$ be an integral curve of $D$ steering $x$ to $\partial A(x)$. Then there is $a 0 \leqq t<a$ and $a$ vector field $X \in D$ such that

$$
X_{s}(\gamma(t)) \in \operatorname{Int} A(x)
$$

for $s>0$. 
Proof. Suppose to the contrary that for any $X \in D$ and all $0 \leqq$ $t<a$,

$$
X_{s}(\gamma(t)) \in \partial A(x) \text {. }
$$

Without loss of generality we can assume that for some $Y \in D$ and $0<\varepsilon<a$

$$
\gamma(t)=Y_{t}(x)
$$

for $0 \leqq t<\varepsilon$. Then

$$
[X, Y](\gamma(t))=a(t) Y(\gamma(t))
$$

for all $0 \leqq t<\varepsilon$. Which implies that

$$
\operatorname{dim} L(\gamma(t))=1
$$

for $0 \leqq t<\varepsilon$ which contradicts the assumptions when $t=0$.

Proposition 10. Let $\operatorname{dim} L(w)=2$ for all points $w \in \partial A(x)$ and let $\gamma:[0, a] \rightarrow \boldsymbol{R}^{2}$ be an integral curve of $D$ steering $x$ to $\partial A(x)$. Let $y=\gamma\left(t_{0}\right)$ with $0<t_{0}<a$. Then there exists a neighborhood $V$ of $y$ such that the set $\partial A(x) \cap V$ is homeomorphic to an interval.

Proof. By Lemma 9 there exist points $z_{1}, z_{2} \in \operatorname{Im} \gamma$ and vector fields $X^{1} \in D, X^{2} \in D_{N}$ with the property that

$$
\begin{array}{ll}
z_{i}=\gamma\left(t_{i}\right) & t_{1}<t_{0}<t_{2}, \\
X_{s}^{1}\left(z_{1}\right) \in \operatorname{Int} A(x) & 0<s \leqq a_{1}, \\
X_{s}^{2}\left(z_{2}\right) \in \operatorname{Int} A_{N}(\gamma(a)) & 0<s \leqq a_{2} .
\end{array}
$$

Define $\gamma_{i}:\left[0, a_{1}\right] \rightarrow R^{2}$ by

$$
\gamma_{i}(s)=X_{s}^{i}\left(z_{i}\right)
$$

for $i=1,2$ and let

$$
L_{i}=\left[0, a_{i}\right] \times\left[0, t_{2}-t_{1}\right] .
$$

Using Lemma 8 with $c_{n}=t_{2}-t_{1}$ and $H_{i}: L_{i} \rightarrow \boldsymbol{R}^{2}$ defined in an obvious way we conclude that $H_{i}\left(L_{i}\right)$ are homeomorphic to rectangles and by Lemma 6 we can see that

$$
H_{1}\left(L_{1}\right) \cap H_{2}\left(L_{2}\right)=\left\{H_{1}(0, t): 0 \leqq t \leqq t_{2}-t_{1}\right\}=\left\{H_{2}(0, t): 0 \leqq t \leqq t_{2}-t_{1}\right\} \text {. }
$$

We conclude that the set

$$
H_{1}\left(L_{1}\right) \cup H_{2}\left(L_{2}\right)
$$

is homeomorphic to a rectangle and that 


$$
y \in \operatorname{Int}\left(H_{1}\left(L_{1}\right) \cup H_{2}\left(L_{2}\right)\right) .
$$

Let $V=\operatorname{Int}\left(H_{1}\left(L_{1}\right) \cup H_{2}\left(L_{2}\right)\right)$. Since $\operatorname{Int}\left(H_{1}\left(L_{1}\right)\right) \leqq \operatorname{Int} A(x)$ and Int $H_{2}\left(L_{2}\right) \subseteq \operatorname{Int} A_{N}(\gamma(a))$,

$$
\partial A(x) \cap\left(\operatorname{Int}\left(H_{1}\left(L_{1}\right)\right) \cup \operatorname{Int}\left(H_{2}\left(L_{2}\right)\right)\right)=\varnothing
$$

it follows that if $w \in \partial A(x) \cap V$ then

$$
w \in\left\{H_{1}(0, t): 0 \leqq t \leqq t_{2}-t_{1}\right\}
$$

which proves the proposition.

THEOREM 11. Let $\operatorname{dim} L(w)=2$ for all $w \in \partial A(x)$ and let $A(x)$ be closed. If the set $\partial A(x)$ is not empty then it is homeomorphic to a Jordan arc or a closed Jordan curve.

Proof. Assume that

$$
\partial A(x) \neq \varnothing .
$$

Let $y \in \partial A(x)$ be a point other than $x$ and let $\gamma$ be an integral curve of $D$ steering $x$ to $y$. Moreover let us assume that $\gamma$ is a homeomorphism from its domain onto its image. Let $\Sigma_{1}$ be the set of all integral curves $\sigma:[0, a(\sigma)] \rightarrow \partial A(x)$ such that $\operatorname{Im} \gamma \cong \operatorname{Im} \sigma$ and $\sigma$ is a homeomorphism onto its image. Let

$$
B_{1}=\bigcup_{\sigma \in \Sigma_{1}} \operatorname{Im} \sigma \text {. }
$$

Clearly $B_{1}$ is connected. Let us introduce an ordering on $B_{1}$ as follows. We will say that

$$
x_{1}<x_{2}
$$

if and only if there exists $\sigma \in \Sigma_{1}$ such that $\sigma$ steers $x$ to $x_{2}$ with $x_{1} \in \operatorname{Im} \sigma$ and $x_{1} \neq x_{2}$. Clearly $x$ is the only minimal element of $B_{1}$. We claim that if there exists a maximal element then it is unique and $B_{1}$ is a homeomorphic image of a closed interval.

Suppose that $z_{i}, i=1,2$ are maximal elements of $B_{1}$. Let $\sigma_{i}:\left[0, a\left(\sigma_{i}\right)\right] \rightarrow \partial A(x)$ be integral curves of $D$ steering $x$ to $z_{i}$. Note that $\operatorname{Im} \sigma_{i}$ are homeomorphic images of closed intervals, thus they are closed and $\operatorname{Im} \sigma_{1} \cap \operatorname{Im} \sigma_{2}$ must contain its maximal element, say $z_{3}$. Then $z_{3}$ would be a "branching" point contradicting Corollary 5 . Thus there is a unique maximal point in $B_{1}$ and the integral curve steering $x$ to this point is a homeomorphism that we were looking for.

Assume that there is no maximal point in $B_{1}$ and that there exists an increasing sequence of points $\left\{x_{n}\right\}_{n=1}^{\infty}$ in $B_{1}$, such that the limit point $y$ of $\left\{x_{n}\right\}_{n=1}^{\infty}$ exists, is an element of $B_{1}$ and is such that 
$y<x_{k}$ for some $k \geqq 1$. We claim that in this case $y=x$ and that $B_{1}=\partial A(x)$ and is homeomorphic to a circle. Indeed if $x_{k-1}<y<$ $x_{k},\left(x_{0}=x\right)$, then we would contradict Proposition 10. Thus if $y<x_{k}$ for some $k$ then $y=x$. We can assume without loss of generality that $x=y=\lim x_{n}$. Let $\gamma_{1}:[0, a] \rightarrow \partial A(x)$ be an integral curve of $D$ steering $x$ to $x_{1}$. Since $x_{1}<x_{2}$ there exists an integral curve $\gamma_{2}^{1}:\left[0, a_{2}^{1}\right] \rightarrow \partial A(x)$ steering $x_{1}$ to $x_{2}$. We can construct now an integral curve of $D, \gamma_{2}:\left[0, a_{1}+a_{2}^{1}\right]=[0, a] \rightarrow \partial A(x)$ steering $x$ to $x_{2}$, with $\operatorname{Im} \gamma_{1}=\gamma_{2}\left(\left[0, a_{1}\right]\right)$, by

$$
\gamma_{2}(t)= \begin{cases}\gamma_{1}(t) & 0 \leqq t \leqq a_{1} \\ \gamma_{2}^{1}\left(t-a_{1}\right) & a_{1} \leqq t \leqq a_{2}\end{cases}
$$

By induction we can define entire sequence of integral curves of $D, \gamma_{n}:\left[0, a_{n}\right] \rightarrow \partial A(x)$ steering $x$ to $x_{n}$ with $\operatorname{Im} \gamma_{n-1}=\gamma_{n}\left(\left[0, a_{n-1}\right]\right)$. Note that $a_{n-1}<a_{n}$. Let

$$
a=\lim _{n \rightarrow \infty} a_{n} \text {. }
$$

If $a<\infty$, let $h:[0, a] \rightarrow S^{1}$ such that

$$
h(0)=h(a)=p^{1}
$$

and $h$ is a continuous map which is a homeomorphism onto its image if restricted to $[0, a)$. Define $H: S^{1} \rightarrow \partial A(x)$ by

$$
H(p)=\gamma_{n}\left(h^{-1}(p)\right)
$$

if $a_{n-1}<h^{-1}(p) \leqq a_{n}$ and

$$
H\left(p^{1}\right)=x .
$$

Clearly $H$ is a homeomorphism. If $a=\infty$, let $k=S^{1} \rightarrow[0, \infty)$ be a continuous $1-1$ mapping defined in such a way that

$$
k(z)=0
$$

for $z=(1,0)$

$$
\lim _{\theta \rightarrow 0^{+}} k\left(e^{i \theta}\right)=0
$$

and

$$
\lim _{\theta \rightarrow 0^{-}} k\left(e^{i \theta}\right)=\infty
$$

Again define $H: S^{1} \rightarrow \partial A(x)$ by

$$
H(p)=\gamma_{n}(k(p))
$$

if $a_{n-1}<k(p) \leqq a_{n}$ and 


$$
H(z)=x \text {. }
$$

Clearly $H$ is a homeomorphism. Thus if there is an increasing sequence $\left\{x_{n}\right\}$ in $B_{1}$ converging to a point $y$ in $B_{1}$ with $y<x_{k}$ for some $k$ then $y=x$ and $B_{1}$ is homeomorphic to $S_{1}$. An argument exactly parallel to one in Proposition 4 will show that there are no integral curves of $D$ steering $x$ to $\partial A(x)$ which are not contained in $B_{1}$. Thus $B_{1}=\partial A(x)$.

Assume now that there is no increasing sequence $\left\{x_{n}\right\}_{n=1}^{\infty}$ in $B_{1}$ such that its limit point $y$ is smaller than $x_{k}$ for any $k=1,2, \cdots$. We claim that $B_{1}$ is homeomorphic to $[0,1)$.

Since $\boldsymbol{R}^{2}$ is a second countable space it follows that $\operatorname{cl} B_{1} \backslash\{x\}$ is a second countable space, hence it is Lindelöf space. It follows from Proposition 10 that for any $y>x$ set

$$
B_{1}(y)=\left\{z \in B_{1}: x<z<y\right\}
$$

is open in the relative topology of the $\mathrm{cl} B_{1}$. Moreover

$$
\operatorname{cl} B_{1} \backslash\{x\}=\bigcup_{y \in B_{1} \backslash\{x\}} B_{1}(y) \text {. }
$$

By the properties of Lindelöf spaces (see Dugundji [1]) there exists a sequence $\left\{y_{n}\right\}_{n=1}$ such that

$$
\operatorname{cl} B_{1} \backslash\{x\}=\bigcup_{n=1}^{\infty} B_{1}\left(y_{n}\right) \text {. }
$$

We can assume without loss of generality that

$$
B_{1}\left(y_{n}\right) \cong B_{1}\left(y_{n+1}\right)
$$

for all $n=1,2, \cdots$. It is easy to see that the sequence $\left\{y_{n}\right\}_{n=1}^{\infty}$ is cofinal in $B_{1}$, i.e., for any element $z$ of $B_{1}$ there exists an $n$ such that $z<y_{n}$. Let $x=y_{0}$ and let $\gamma_{n}^{1}:\left[0, a_{n}\right] \rightarrow \partial A(x)$ be an integral curve of $D$ steering $y_{n}$ to $y_{n+1}$. Define $\gamma_{n}:\left[0, b_{n}\right] \rightarrow \partial A(x)$ where $b_{n}=$ $\sum_{k=0}^{n-1} \alpha_{k}$ by

$$
\gamma_{n}(t)=\gamma_{i}^{1}\left(t-\sum_{k=0}^{i} a_{k}\right)
$$

for $\sum_{k=0}^{i} a_{k} \leqq t \leqq \sum_{k=0}^{i+1} a_{k}$. It is clear that $\gamma_{n}$ is an integral curve of $D$ steering $x$ to $b_{n}$. Let $b=\lim _{n \rightarrow \infty} b_{n}$. Define $H:[0, b) \rightarrow \partial A(x)$ by

$$
H(t)=\gamma_{n}(t)
$$

for $t \leqq b_{n}$. It is clear that $H$ is well defined, one-to-one, onto and continuous. We will show that $H^{-1}$ is also a continuous map. Let $\left\{z_{n}\right\}_{n=1}^{\infty}$ be a sequence in $B_{1}$ such that $\lim _{n \rightarrow \infty} z_{n}=z_{0} \in B_{1}$. Since $\left\{y_{n}\right\}_{n=1}^{\infty}$ is cofinal there exists an $N$ such that, except for finitely many, $z_{n} \in B_{1}\left(y_{N}\right)$. Note that $\gamma_{N}:\left[0, b_{N}\right] \rightarrow \partial A(x)$ is a homeomorphism onto 
its image and that

$$
B_{1}\left(y_{N}\right) \cong \operatorname{Im} \gamma_{N}
$$

Thus

$$
\lim _{n \rightarrow \infty} H^{-1}\left(z_{n}\right)=\lim _{n \rightarrow \infty} \gamma_{N}^{-1}\left(z_{n}\right)=\gamma_{N}^{-1}\left(z_{0}\right)=H^{-1}\left(z_{0}\right) .
$$

This proves that $H$ is a homeomorphism.

We have thus shown that $B_{1}$ is homeomorphic to one of the following objects: closed interval, half closed interval or a circle. In case of a circle we have shown that $B_{1}=\partial A(x)$.

Assume that $B_{1} \neq \varnothing$. If there is an integral curve of $D$ steering $x$ to $A(x) \backslash B_{1}$, then we can construct $B_{2}$ in the same manner as we constructed $B_{1}$. Following the proof for $B_{1}$ we establish that $B_{2}$ must be homeomorphic to a closed or a half closed interval. It could not be homeomorphic to circle for then $B_{2}=\partial A(x)$ and $B_{1}=\varnothing$. Clearly

$$
B_{1} \cup B_{2}=\partial A(x) .
$$

Thus in general the following three cases may occur

(1) $B_{1}=\partial A(x), B_{2}=\varnothing$

(2) $B_{1} \neq \varnothing ; B_{2} \neq \varnothing$

$$
\begin{aligned}
& \{x\}=\operatorname{cl} B_{1} \cap \operatorname{cl} B_{2} \\
& \{x\}=\operatorname{cl} B_{1} \cap \operatorname{cl} B_{2} .
\end{aligned}
$$

We have already investigated case (1). If case 2 (a) occurs it is clear that $\partial A(x)$ is homeomorphic to a Jordan arc. For case $2(b)$ we will show that $\partial A(x)$ is homeomorphic to $S^{1}$ by showing that at least one of $B_{1}$ and $B_{2}$ is closed, say $B_{1}=\gamma[0,1]$ with $\gamma(0)=x$, and that there is at most one point distinct from $x$ in $\mathrm{cl} B_{1} \cap \mathrm{cl} B_{2}$ namely $\gamma(1)$.

Suppose $y_{1}, y_{2} \in \operatorname{cl} B_{1} \cap \operatorname{cl} B_{2}$ and $y_{i} \neq x$. Note that both $y_{1}$ and $y_{2}$ are elements of $B_{1} \cup B_{2}=\partial A(x)$ as $A(x)$ is closed. Assume that $y_{1}, y_{2} \in B_{1}$. Since $B_{1}$ is a Jordan arc there is an induced order on $B_{1}$ and one of them, say $y_{1}$, is a point in between $x$ and $y_{2}$. This contradicts Proposition 10. Suppose that $y_{1} \in B_{1}$ and $y_{2} \in B_{2}$. By Proposition 10 none of the two can be an internal point of $B_{i} . \quad B_{i} \neq \varnothing, i=1,2$, and none is homeomorphic to a circle, thus $B_{i}$ is homeomorphic to a half-open interval or a closed interval, but in the former case the only point which is not internal point of $B_{i}$ is $x$. Hence $B_{i}, i=1,2$ is homeomorphic to a closed interval.

Since $A(x)$ is closed and $\partial A(x)=B_{1} \cup B_{2}$ it follows that $y_{1}=y_{2}$.

TheOREM 12. Let $\operatorname{dim} L(w)=2$ for all $w \in \partial A(x)$. Then set $A(x)$ 
is homeomophic to one of the following objects

(i ) closed half plane

(ii) closed disk

(iii) complement of an open disk

(iv) $\boldsymbol{R}^{2}$.

Proof. Suppose that $\partial A(x)$ is homeomorphic to an open interval. Let $\gamma:(0,1) \rightarrow \partial A(x)$ be the homeomorphism. Consider

$$
\Pi: S^{2} \longrightarrow R^{2}
$$

the stereographic projection. Assume that point $p$ in $S^{2}$ is such that $\Pi_{R}$ restricted to $S^{2} \backslash\{p\}$ is a homeomorphism. It is clear that $\Pi_{R}^{-1}$ is a homeomorphism and that

$$
p=\lim _{t \rightarrow 1^{-}} \Pi_{R}^{-1} \gamma(t)=\lim _{t \rightarrow 0^{+}} \Pi_{R}^{-1} \gamma(t) .
$$

Thus $\Pi_{R}^{-1} \gamma(0,1) \cup\{p\}$ is homeomorphic to $S^{1}$ which separates $S^{2}$ into two components each of which is homeomorphic to an open two dimensional disk. Since $S^{2} \backslash\{p\}$ is homeomorphic to $\boldsymbol{R}^{2}$ via $\Pi$ one of these components must be $\Pi_{R}^{-1}\left(\boldsymbol{R}^{2} \backslash A(x)\right)$ and the other $\Pi_{R}^{-1}(\operatorname{Int} A(x))$. Thus $\Pi_{R}^{-1}(A(x)) \cup\{p\}$ is homeomorphic to a closed disk, and $\Pi_{R}^{-1}(A(x))$ is homeomorphic to a closed half plane.

Suppose that $\partial A(x)$ is homeomorphic to $S^{1}$. Then by Jordan curve theorem $\boldsymbol{R}^{2} \mid \partial A(x)$ has two components, one of which must be Int $A(x)$. If this is the bounded one then $A(x)$ is homeomorphic to a closed disk, otherwise $\boldsymbol{R}^{2} \backslash A(x)$ is homeomorphic to an open disk. If $\partial A(x)$ is an empty set then $A(x)=\boldsymbol{R}^{2}$. Indeed the only subsets of $\boldsymbol{R}^{2}$ with empty boundary are empty set and $\boldsymbol{R}^{2}$. Set $A(x)$ is nonempty thus $A(x)=R^{2}$.

THEOREM 13. Let $\partial A(x) \neq \varnothing$. Let $\operatorname{dim} L(w)=2$ for all $w \in \partial A(x)$. Let $B(x)$ denote the set of points $z \in \partial A(x)$ such that there exists an integral curve of $D, \gamma:[0, a] \rightarrow \partial A(x)$ and $a t_{1}$ in $(0, a)$, with $z=\gamma\left(t_{1}\right)$. Then $B(x)$ is an imbedded $C^{1}$-submanifold of $\boldsymbol{R}^{2}$ with at most two connected components.

Proof. Set $B(x)$ is contained in $\partial A(x) \cap A(x)$. Proposition 10 shows that $B(x)$ is an imbedded $C^{0}$-manifold. Let $z \in B(x)$ and $\gamma$ be as above, determined by piecewise constant control $u:[0, a] \rightarrow D$. We have only to consider the case when $t_{1}$ is a point of discontinuity of $u$. Let $X^{i}, X^{j} \in D$ be two distinct values of $u$ near $t_{1}$ and assume that the integral curves of $X^{i}$ and $X^{j}$ are transversal at $z$. Then one of the integral curves steers either an interior point of $A(x)$ to its boundary or a point of $\partial A(x) \cap A(x)$ to the exterior of $A(x)$, which 
is impossible. It follows from our previous discussion that if $\operatorname{dim} L(w)=2$ for all $w \in \partial A(x)$ then $\partial A(x) \backslash B(x)$ contains at most two points.

From the topological point of view the boundary of a closed set of attainability has been completely described, however from the control theoretic point of view it seems necessary to subdivide the three cases described in Theorem 11 into the following:

I. The boundary of $A(x)$ is homeomorphic to an open interval.

II. The boundary of $A(x)$ is homeomorphic to a circle.

(1) There are no two distinct integral curves of $D$ steering $x$ to $\partial A(x)$.

(a) There exists a nonconstant integral curve of $D, \gamma:[0, a] \rightarrow$ $\partial A(x)$ steering $x$ to $x$ with $\gamma(a)=x$.

(b) There is no integral curve having the above properties.

(2) There are two distinct nonconstant integral curves of $D$ steering $x$ to $\partial A(x)$.

(a) There exists $y \in \partial A(x)$ and two distinct nonconstant curves $\gamma_{1}$ and $\gamma_{2}$ of $D$ steering $x$ to $y$.

(b) There is no point $y$ in $\partial A(x)$ having the above properties.

III. The boundary of $A(x)$ is empty.

Let us note that in case I we must have two distinct nonconstant integral curves of $D$ steering $x$ to $\partial A(x)$.

All the possible cases listed above actually occur. We illustrate all of them in the examples below, omitting some of the lengthy computations. In each figure the shaded area represents the set of attainability from the point indicated by its coordinates.

ExAmple 14. 1. Let $D=\left\{X_{1}, X_{2}, 0\right\}$ with

$$
\begin{array}{ll}
X_{1}\left(x_{1}, x_{2}\right)=\left(-2 x_{2}, x_{1}+2 x_{2}\right) & \text { for } \quad\left(x_{1}, x_{2}\right) \in \boldsymbol{R}^{2}, \\
X_{2}\left(x_{1}, x_{2}\right)=\left(-2 x_{2}, x_{1}+2 x_{2}+e^{\pi}-1\right) & \text { for } \quad\left(x_{1}, x_{2}\right) \in \boldsymbol{R}^{2} .
\end{array}
$$

Then the set $A\left(x_{1}, 0\right)$ is equal to $\boldsymbol{R}^{2}$ if $0 \leqq x_{1}<1$, is of type IIIa if $x_{1}=1$, is of type II2a if $1<x_{1}<1+e^{-\pi}$ and is of type I if $x_{1} \geqq 1+e^{-\pi}$. (See Fig. 1.)

2. Let $D=\left\{X_{1}, X_{2}, 0\right\}$ where

$$
\begin{array}{ll}
X_{1}\left(x_{1}, x_{2}\right)=\left(-x_{1},-x_{2}\right) & \text { for } \quad\left(x_{1}, x_{2}\right) \in R^{2}, \\
X_{2}\left(x_{1}, x_{2}\right)=\left(2 x_{2},-x_{1}+2 x_{2}-1\right) & \text { for } \quad\left(x_{1}, x_{2}\right) \in R^{2} .
\end{array}
$$

Then the set $A\left(-e^{\pi}-1,0\right)$ is of type II2b. (See Fig. 2.)

3. Let $D=\left\{X_{1}, X_{2}, 0\right\}$ with

$$
\begin{array}{ll}
X_{1}\left(x_{1}, x_{2}\right)=\left(-x_{1},-x_{2}\right) & \text { for } \quad\left(x_{1}, x_{2}\right) \in \boldsymbol{R}^{2}, \\
X_{2}\left(x_{1}, x_{2}\right)=\left(-2 x_{1}-2 x_{2}-2, x_{1}+1\right) & \text { for } \quad\left(x_{1}, x_{2}\right) \in \boldsymbol{R}^{2} .
\end{array}
$$



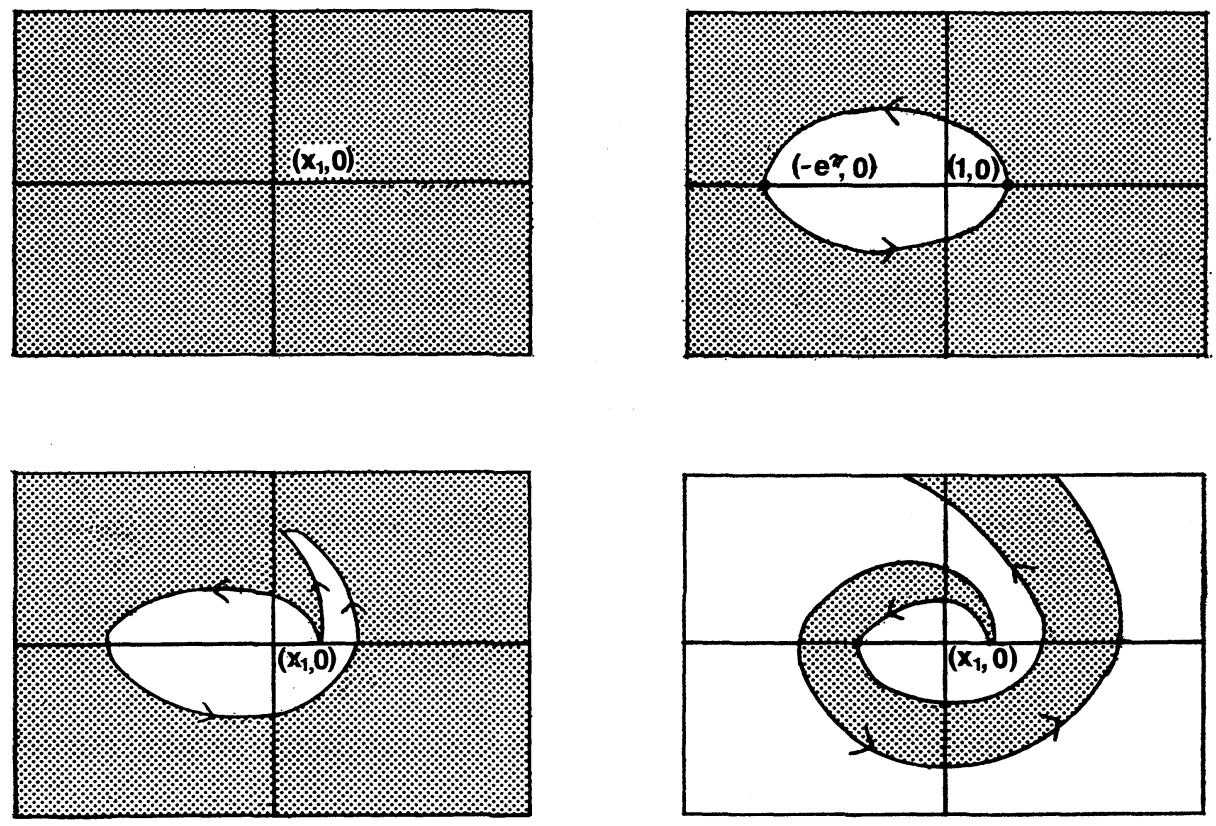

Figure 1

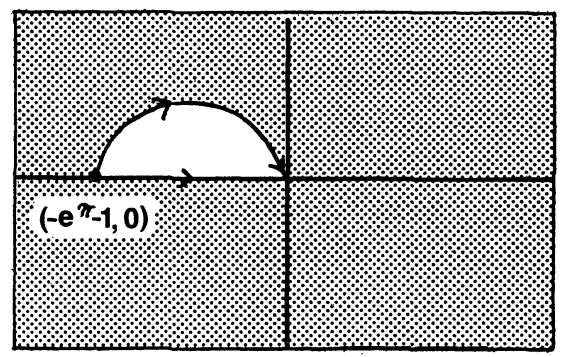

FIGURE 2

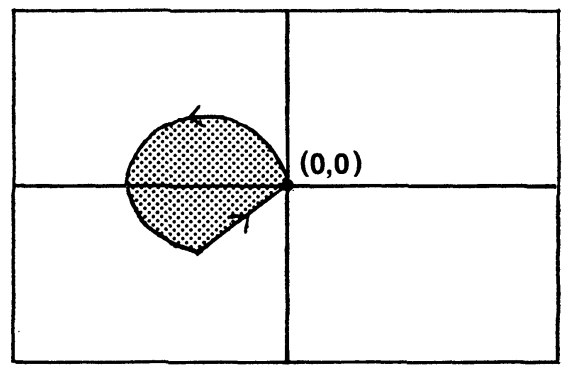

FiguRe 3

Then the set $A(0,0)$ is of type IIb. (See Fig. 3.)

ACKNowledgment. I want to express my deep gratitude to Professor F. Albrecht for his advice and moral support. 


\section{REFERENCES}

1. J. Dugundji, Topology, Allyn and Bacon, 1969.

2. Y. Gerbier, Classification de couple de systems dynamique du plan: Application a la theorie de la commande, These, L'Universite de Bordeaux 1, 1974.

3. J. Gronski, Perturbations of open sets of attainability, Mathematical Systems Theory, 10 (1976).

4. A. Krener, A Generalization of Chow's theorem and the Bang-bang theorem to nonlinear control problems, SIAM J. Control, 12 (1974), 43-52.

5. C. Lobry, Quelques aspects qualitative de la theorie de la commande, These, L'Universite de Grenoble, 1972.

6. E. Roxin, A Geometric Interpretation of Pontryagin's Maximum Principle, Nonlinear Differential Equations and Nonlinear Mechanics, Academic Press, New York, 1963.

Received May 16, 1977.

Cleveland State University

Cleveland, OH 44115 



\section{PACIFIC JOURNAL OF MATHEMATICS}

\section{EDITORS}

RICHARD ARENS (Managing Editor)

University of California

Los Angeles, California 90024

C. W. Curtis

University of Oregon

Eugene, OR 97403

C. C. MOORE

University of California

Berkeley, CA 94720

\section{J. DUGUNDJI}

Department of Mathematics University of Southern California Los Angeles, California 90007

R. Finn AND J. Milgram Stanford University Stanford, California 94305

\section{ASSOCIATE EDITORS}

E. F. BeCK ENBACH

B. H. NeUMaNN

F. WOLF

K. Yoshida

\section{SUPPORTING INSTITUTIONS}

UNIVERSITY OF BRITISH COLUMBIA CALIFORNIA INSTITUTE OF TECHNOLOGY UNIVERSITY OF CALIFORNIA MONTANA STATE UNIVERSITY UNIVERSITY OF NEVADA, RENO NEW MEXICO STATE UNIVERSITY OREGON STATE UNIVERSITY UNIVERSITY OF OREGON
UNIVERSITY OF SOUTHERN CALIFORNIA STANFORD UNIVERSITY UNIVERSITY OF HAWAII UNIVERSITY OF TOKYO UNIVERSITY OF UTAH WASHINGTON STATE UNIVERSITY UNIVERSITY OF WASHINGTON 


\section{Pacific Journal of Mathematics \\ Vol. 77, No. $1 \quad$ January, 1978}

Dan Amir, Chebyshev centers and uniform convexity ............... 1

Lawrence Wasson Baggett, Representations of the Mautner group. I ..... 7

George Benke, Trigonometric approximation theory in compact totally

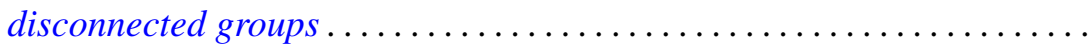

M. Bianchini, O. W. Paques and M. C. Zaine, On the strong compact-ported topology for spaces of holomorphic mappings ..................

Marilyn Breen, Sets with $(d-2)$-dimensional kernels

J. L. Brenner and Allen Kenneth Charnow, Free semigroups of $2 \times 2$

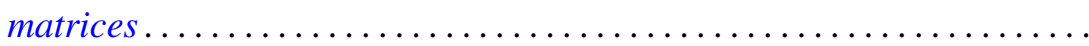

David Bressoud, A new family of partition identities .................

David Fleming Dawson, Summability of matrix transforms of stretchings

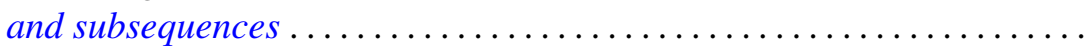

Harold George Diamond and Paul Erdôs, A measure of the nonmonotonicity

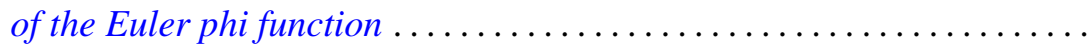

Gary Doyle Faulkner and Ronald Wesley Shonkwiler, Kernel dilation in reproducing kernel Hilbert space and its application to moment

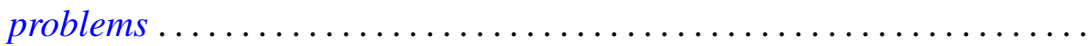

Jan Maksymilian Gronski, Classification of closed sets of attainability in the

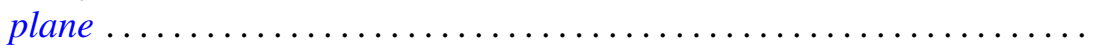

H. B. Hamilton and T. E. Nordahl, Semigroups whose lattice of congruences

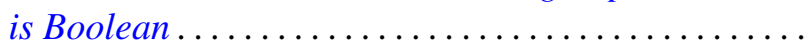

Harvey Bayard Keynes and D. Newton, Minimal $(G, \tau)$-extensions ...

Anthony To-Ming Lau, The Fourier-Stieltjes algebra of a topological

semigroup with involution.

B. C. Oltikar and Luis Ribes, On prosupersolvable groups ...

Brian Lee Peterson, Extensions of pro-affine algebraic groups ...

Thomas M. Phillips, Primitive extensions of Aronszajn spaces ...

Mehdi Radjabalipour, Equivalence of decomposable and 2-decomposable operators. .

M. Satyanarayana, Naturally totally ordered semigroups .

Fred Rex Sinal, A homeomorphism classification of wildly imbedded two-spheres in $S^{3}$

Hugh C. Williams, Some properties of a special set of recurring

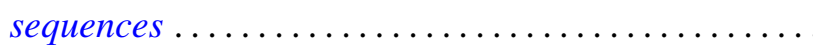

